\title{
Inflammatory Verrucous Epidermal Nevus: Treated with Surgical Excision
}

\author{
Authors \\ Ravinder Singh ${ }^{1}$, Abhishek Thakur ${ }^{2}$, Shikha Verma ${ }^{3^{*}}$ \\ ${ }^{1}$ Medical Officer, Dermatology DR RKGMC Hamirpur H.P India \\ ${ }^{2}$ Senior Resident, Department of Dermatology, Dr RKGMC Hamirpur H.P India \\ ${ }^{3}$ Senior Resident, Department of Pediatrics Dr RPGMC Kangra H.P India \\ *Corresponding Author \\ Shikha Verma
}

\begin{abstract}
The "inflammatory linear verrucous epidermal nevus" is a rare disease, consisting of hyperplasia of the normal components of the epidermis typically present at birth, but can occur anytime during childhood and may rarely appear in adulthood. Its clinical features include erythematous and hyperkeratotic, warty, sometimes psoriasiform or lichenoid patches with a typical linear arrangement. They usually occur on a limb following the lines of Blaschko, more rarely on the trunk, in a curvilinear pattern, or in different areas. We present a 17 years old female with inflammatory linear verrucous epidermal nevus on right side of her abdomen.
\end{abstract}

\section{Introduction}

Epidermal nevi are defined as congenital malformations or hamartomas derived from embryonic ectoderm. It is typically seen at birth or develops in early childhood and evolves until puberty $^{[1]}$. Linear verrucous epidermal nevi clinically appear as verrucous papules and plaques following Blaschko's lines distributed in a linear pattern. Inflammatory linear verrucous epidermal nevus (ILVEN) is a rare variant of epidermal verrucous nevus that commonly affects females. Prevalence of linear verrucous epidermal nevus is estimated to be 1:1000 live births. This condition is characterized by the appearance of recurrent inflammatory phenomena, with chronic eczematous or psoriasiform aspects, usually unilateral, with severe pruritus, and refractory to therapy ${ }^{[2,3]}$.

\section{Case Report}

A 17 years old female presented in dermatology OPD with erythematous warty plaques over right side of anterior abdominal wall extending form right lateral aspect toward the umbilicus with area of normal skin in between. The patient was born to non-consanguineous parentage, with normal birth and developmental history. Past and family history was unremarkable. Systemic review including skeletal, ophthalmic, and neurological survey was non-contributory. These lesions were present since birth, which started as discrete hyper pigmented papules that gradually increase in size. These lesions were asymptomatic and faintly visible for few years. After age of 11-12 years these lesion increase in size and were associated with pruritus. There was history of application various topical medications in the past but no improvement. When 
patient present to us skin biopsy from representative lesions was done. Histology showed hyperkeratosis, acanthosis and papillomatosis and dense chronic inflammatory infiltrate in the dermis. On the basis of history, clinical findings, and histologic features a diagnosis of "ILVEN was made. She was initially treated with 2-3 sessions of electocautry but the results were not satisfactory and finally excision was done.

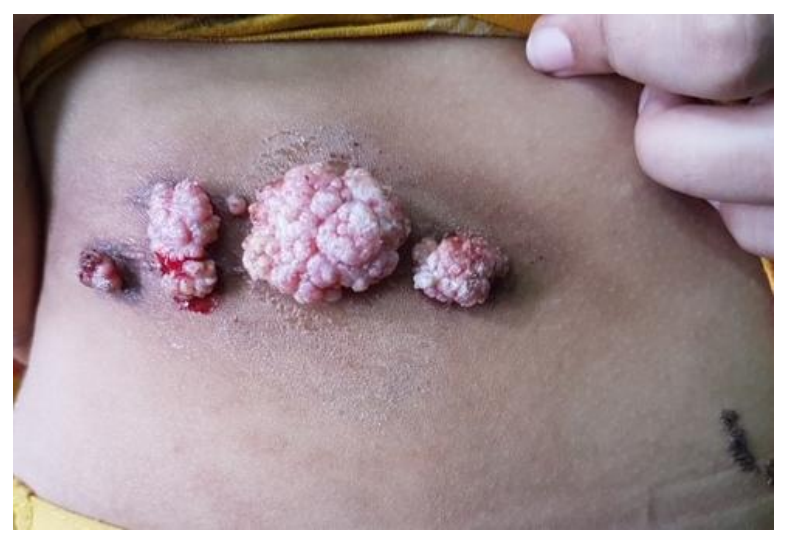

Figure 1: Erythematous, hyperkertotic, warty plaque over anterior abdominal wall

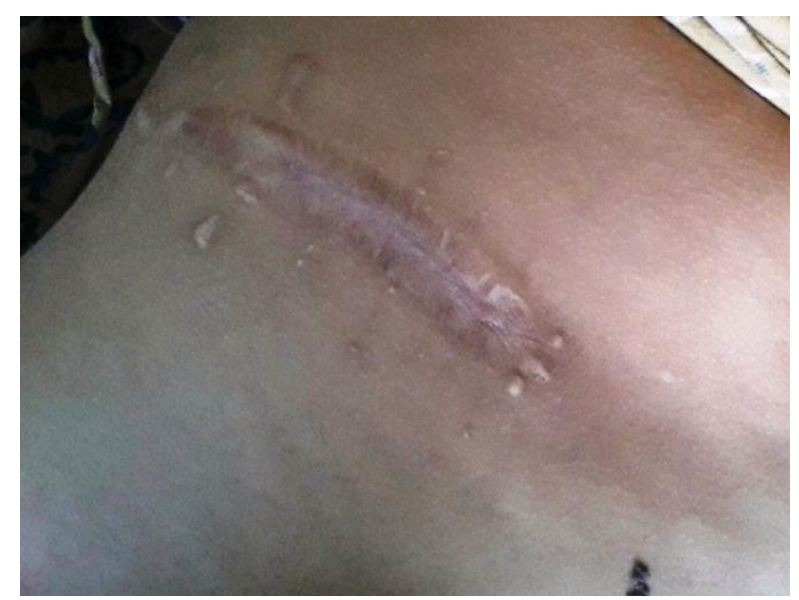

Figure 2 Surgical excision of lesion

\section{Discussion}

An epidermal nevus is an abnormal, benign lesion caused by an overgrowth of skin cells. It is typically seen at birth or develops in early childhood and evolves until puberty. They are characterized by hyperplasia of the epidermis and adnexal structures and may be associated with serious disfiguration ${ }^{[4]}$. They can be divided into either keratinocytic types (nonorganoid) or organoid types characterized by hyperplasia of adnexal structures, such as sebaceous glands, sweat glands and hair follicles. Keratinocytic tumours differ according to the distribution of the lesions or the predominant histologic cell type: keratinocyte (Verrucous epidermal nevus), sebaceous gland (nevus sebaceous), pilosebaceous unit (nevus comedonicus), eccrine gland (eccrine nevus), or apocrine gland (apocrine nevus) ${ }^{[5,6]}$. The most common type of epidermal nevi is the keratinocytic nevi, also called verrucous epidermal nevi. They are usually present at birth or infancy and may enlarge slowly during childhood. The nevus typically appears as Verrucous papules that coalesce to form well-demarcated, skin coloured, brownish or graybrown, papillomatous plaques ${ }^{[7,8]}$. Altman and Mehregan in 1971 describe inflammatory linear verrucous epidermal nevus as a distinct entity in 25 patients. They coined the term ILVEN labelling it a clinical and histopathologic type of linear verrucous nevus that is often inflammatory or psoriasiform. ILVEN accounts for approximately $5 \%$ of patients with epidermal nevi. ILVEN is caused by somatic mutations that result in genetic mosaicism and, although its physiopathology is still unclear, it is believed that it may be associated with an increase in the production of interleukins 1 and 6 , tumour necrosis factor-alpha, and intercellular adhesion molecule 1. Clinically, it presents with erythematous and verrucous papules with intense pruritus and linear distribution following Blaschko's lines. Although cases of bilateral involvement have been described, the disease is often unilateral and localized to one extremity. The classical clinical criteria for diagnosis of this condition was suggested by Altman and Mehregan in $1971^{[9]}$ and later modified by Morag and Metzker in $1985^{[10]}$ viz. early age of onset, predominance in females, frequent involvement of the left leg, pruritus, distinctive psoriasiform appearance, and marked refractoriness to therapy. The histopathologic features of epidermal nevi include moderate hyperkeratosis, acanthosis, papillomatosis in psoriasiform pattern, and dermal infiltration or even Munro's micro-abscesses. The rete ridges are elongated, and in some cases focal thickening of the 
granular layer and columns of parakeratosis are seen ${ }^{[11]}$. In our case lesions were present since birth associated with pruritus and typical histopathologic findings. The differential diagnosis of ILVEN must be done with various dermatoses, such as other epidermal nevi, Darier's disease, linear porokeratosis, linear lichen planus, linear psoriasis, and lichen striatus ${ }^{[12]}$. The treatment of epidermal nevi is challenging. Multiple medical and surgical treatments have been attempted, but no ideal or universally acceptable treatment has emerged. Many therapies have been attempted for the epidermal nevi including intralesional as well as topical steroids, topical and systemic retinoids, topical 5fluorouracil, podophyllin, dermabrasion, cryosurgery, and excisional surgery. However, these treatment options tend to either fall short of complete eradication of the nevus or result in an unacceptable scar that can be as disfiguring as the nevus itself. Variable results with laser treatment of epidermal nevi including Argon, Er: YAG, pulsed dye, and $\mathrm{CO} 2$ laser have been demonstrated in the past. Recent developments in $\mathrm{CO} 2$ laser technology have made the modern pulsed and scanned $\mathrm{CO} 2$ laser as an excellent treatment of choice for the patients with epidermal nevi ${ }^{[13]}$.

\section{References}

1. Brandling-Bennett HA (University of Washington, Seattle Children's Hospital, Seattle, WA 98105, USA), Morel KD. Epidermal nevi. Pediatr Clin North Am 2010 Oct;57(5):1177- 1198.

2. Solomon LM, Fretzin DF, Dewald RL. The epidermal nevus syndrome. Arch Dermatol 1968;97:273-85.

3. 6. Gon AS, Minelli L, Franzon PG. A case for diagnosis. Ann Bras Dermatol 2010;85:729-31.

4. Panagiotopoulos A (3rd Dermatology Clinic, Andreas Sygros Hospital for Skin Diseases, Athens, Greece.), Chasapi V, Nikolaou V, Stavropoulos P, Kafouros K, Petridis A, Katsambas A. Assessment of cryotherapy for the treatment of Verrucous Epidermal naevi. Acta Derm Venereol 2009;89(3):292294.

5. Hafner C (Department of Dermatology, University of Regensburg, Germany. christian.hafner@klinik.uni-regensburg. de), Vanoers J, Vogt T, Landthaler M, Stoehr R, Blaszyk H, Hofstaedter F, Zwarthoff E, Hartmann A. Mosaicism of activating FGFR3 mutations in human skin causes epidermal nevi. J Clin Invest 2006 Aug;116(8):2201-2207.

6. Cassetty CT (Ronald O. Perelman Department of Dermatology, New York University, USA), Leonard AL. Epidermal nevus. Dermatol Online J 2003 Oct;9(4):43.

7. Levenberg ME, Ermolovich T, Purcell SM. Management of epidermal nevi in the setting of epidermal syndrome. JAOCD 2006;6:5861.

8. Kim JJ (Department of Dermatology, Henry Ford Health System, Detroit, MI 48202, USA), Chang MW, Shwayder T. Topical tretinoin and 5-fluorouracil in the treatment of linear verrucousepi- dermal nevus. J Am Acad Dermatol 2000 Jul;43(1 Pt 1):129-132.

9. Altman J, Mehregan AH. Inflammatory linear verrucosae epidermal nevus. Arch Dermatol 1971;104:385-9.

10. Morag C, Metzker A. Inflammatory linear verrucous epidermal nevus: Report of seven new cases and review of literature. Pediatr Dermatol 1985;3:15-8.

11. Gorlin RJ, Cohen MM, Stefen LL (Epidermal nevus syndrome). In: Syndromes of the head and neck, 3rd ed. New York: Oxford University Press; 1990. p. 362-6.

12. Wolff K, Goldsmith LA, Katz SI, Gilchrest BA, Paller AS, Leffell DJ. Epidermal nevus. In: Fitzpatrick's Dermatology in general medicine, Vol. 1, 5th ed. New York: McGraw Hill, Inc.; 1999. p. 876-8.

13. Boyce S, Alster TS. CO2 Laser treatment of epidermal nevi: Long term success. DermatolSurg 2002;28:611-4. 